\title{
BALANCED COHEN-MACAULAY COMPLEXES
}

\author{
BY \\ RICHARD P. STANLEY ${ }^{1}$
}

\begin{abstract}
ABstracr. A balanced complex of type $\left(a_{1}, \ldots, a_{m}\right)$ is a finite pure simplicial complex $\Delta$ together with an ordered partition $\left(V_{1}, \ldots, V_{m}\right)$ of the vertices of $\Delta$ such that card $\left(V_{i} \cap F\right)=a_{i}$ for every maximal face $F$ of $\Delta$. If $\mathbf{b}=\left(b_{1}, \ldots, b_{m}\right)$, then define $f_{\mathbf{b}}(\Delta)$ to be the number of $F \in \Delta$ satisfying $\operatorname{card}\left(V_{i} \cap F\right)=b_{i}$. The formal properties of the numbers $f_{b}(\Delta)$ are investigated in analogy to the $f$-vector of an arbitrary simplicial complex. For a special class of balanced complexes known as balanced Cohen-Macaulay complexes, simple techniques from commutative algebra lead to very strong conditions on the numbers $f_{b}(\Delta)$. For a certain complex $\Delta(P)$ coming from a poset $P$, our results are intimately related to properties of the Möbius function of $P$.
\end{abstract}

1. Introduction. We are concerned with the problem of obtaining information on the number $f_{i}=f_{i}(\Delta)$ of $i$-dimensional faces of a finite simplicial complex $\Delta$. (All terminology is defined below.) There are two significant classes of complexes $\Delta$ for which a complete characterization of the numbers $f_{i}, 0 \leqslant i \leqslant \operatorname{dim} \Delta$, has been obtained, viz., the class of all complexes and the class of Cohen-Macaulay complexes. Here we introduce a new class which we call balanced complexes. Balanced complexes possess invariants $f_{\mathbf{b}}$ more discriminating than the numbers $f_{i}$, and the formal properties of these invariants will be investigated. In the case of balanced Cohen-Macaulay complexes $\Delta$, simple techniques from commutative algebra lead to conditions on the invariants $f_{\mathbf{b}}$ which are considerably stronger than those obtained merely by assuming $\Delta$ is Cohen-Macaulay. For a certain complex $\Delta(P)$ coming from a poset $P$, our results are intimately related to properties of the Möbius function of $P$.

We now proceed to the basic definitions and terminology. We employ the following notation throughout:

$\mathbf{N}$, set of nonnegative integers,

$\mathbf{P}$, set of positive integers,

$[n],\{1,2, \ldots, n\}$, where $n \in \mathbf{P}$,

Received by the editors July 22, 1977.

AMS (MOS) subject classifications (1970). Primary 05A99; Secondary 06A10, 13H10, 57C05.

$K e y$ words and phrases. Simplicial complex, $f$-vector, $h$-vector, Coehen-Macaulay ring, Hilbert function, Poincaré series, poset, Möbius function.

${ }^{1}$ Partially supported by NSF Grant \# MCS 7308445-A04.

(C) 1979 American Mathematical Society 0002-9947/79/0000-0155/05.75 
$T \subset S, T$ is a subset of $S$, allowing $T=\varnothing$ or $T=S$,

$\mathbf{e}_{i}$, the $i$ th unit coordinate vector in $\mathbf{N}^{m}$, i.e., $\mathbf{e}_{i}=\left(\varepsilon_{1}, \ldots, \varepsilon_{m}\right)$, where $\varepsilon_{j}=\delta_{i j}$.

Now let $\Delta$ be a simplicial complex, or complex for short, on the vertex set $V=\left\{x_{1}, \ldots, x_{n}\right\}$. Thus $\Delta$ is a collection of subsets of $V$ satisfying the two conditions: (i) $\{x\} \in \Delta$ for all $x \in V$, and (ii) if $F \in \Delta$ and $G \subset F$, then $G \in \Delta$. If $F \in \Delta$ and $F$ has $i+1$ elements, then we call $F$ an $i$-face of $\Delta$ and write $\operatorname{dim} F=i$. If $\delta=\max \{\operatorname{dim} F: F \in \Delta\}$, then we call $\Delta$ a $\delta$-complex and write $\operatorname{dim} \Delta=\delta$. If every maximal face of $\Delta$ is a $\delta$-face, then we say that $\Delta$ is pure. Let $f_{i}=f_{i}(\Delta)$ denote the number of $i$-faces of $\Delta$. Thus $f_{0}=n$. The vector $\mathbf{f}=\mathbf{f}(\Delta)=\left(f_{0}, f_{1}, \ldots, f_{\delta}\right)$ is called the $f$-vector of $\Delta$. We will employ the notation $\Delta=\langle a b c, b c d, b d e\rangle$ to indicate that the maximal faces of $\Delta$ are $\{a, b, c\},\{b, c, d\}$ and $\{b, d, e\}$. Hence the $f$-vector of this $\Delta$ is $(5,7,3)$.

The problem often arises of obtaining information about the $f$-vectors of various complexes $\Delta$. The first significant result along these lines, essentially due to Joseph Kruskal [12] and G. Katona [11] (see [9, §8] for an exposition), is an explicit characterization of those vectors $\mathbf{f}=\left(f_{0}, f_{1}, \ldots, f_{\delta}\right)$ which are the $f$-vectors of some complex $\Delta$. We will call such vectors $K$-vectors. Kruskal and Katona actually only proved that the condition in Theorem 1.1 below is necessary; but the sufficiency of this condition is immediate from their proofs.

1.1 THEOREM. Given positive integers $f$ and $i$, write

$$
f=\left(\begin{array}{c}
n_{i} \\
i
\end{array}\right)+\left(\begin{array}{c}
n_{i-1} \\
i-1
\end{array}\right)+\cdots+\left(\begin{array}{c}
n_{j} \\
j
\end{array}\right),
$$

where $n_{i}>n_{i-1}>\cdots>n_{j} \geqslant j \geqslant 1$ (such a representation exists and is unique), and define

$$
f^{(i)}=\left(\begin{array}{c}
n_{i} \\
i+1
\end{array}\right)+\left(\begin{array}{c}
n_{i-1} \\
i
\end{array}\right)+\cdots+\left(\begin{array}{c}
n_{j} \\
j+1
\end{array}\right) .
$$

Then the vector $\mathbf{f}=\left(f_{0}, f_{1}, \ldots, f_{\delta}\right)$ of positive integers is a $K$-vector if and only if $f_{i+1} \leqslant f_{i}^{(i+1)}$ for $0 \leqslant i \leqslant \delta-1$.

One can now ask for special classes of complexes whose $f$-vectors have characterizations analogous to that of Theorem 1.1. One class for which such a characterization can be given consists of the so-called Cohen-Macaulay complexes [23]. These may be defined either algebraically or topologically; it is a basic result of G. Reisner [16] that the two definitions are equivalent. Both definitions will be of use to us here. First we define the notion of a Cohen-Macaulay ring in the case of interest to us. Let $k$ be a field, and let $m$ be a positive integer. Suppose $R$ is a finitely-generated $\mathbf{N}^{m}$-graded $k$-algebra. I.e., the additive group of $R$ can be written as a direct $\operatorname{sum} R=\Sigma_{\alpha \in \mathrm{N}^{m}} R_{\alpha}$, 
where $R_{\alpha} R_{\beta} \subset R_{\alpha+\beta}, R_{0}=k$, and $R$ is finitely generated as a $k$-algebra. If $x \in R_{\alpha}$ we say that $x$ is homogeneous of degree $\alpha$, written $\operatorname{deg} x=\alpha$. If we wish to emphasize that $\alpha \in \mathbf{N}^{m}$, then we say that $x$ is $\mathbf{N}^{m}$-homogeneous. It follows from the fact that $R$ is finitely-generated that $\operatorname{dim}_{k} R_{\alpha}<\infty$ for each $\alpha \in \mathbf{N}^{m}$, and we define the Hilbert function $H(R, \alpha)=\operatorname{dim}_{k} R_{\alpha}, \alpha \in \mathbf{N}^{m}$. If $\alpha=\left(\alpha_{1}, \ldots, \alpha_{m}\right)$ let $\lambda^{\alpha}=\lambda_{1}^{\alpha_{1}} \cdots \lambda_{m}^{\alpha_{m}}$, and define the Poincaré series $F(R, \lambda)=\Sigma_{\alpha} H(R, \alpha) \lambda^{\alpha}$. It is well known that the formal power series $F(R, \lambda)$ represents a rational function of $\lambda=\left(\lambda_{1}, \ldots, \lambda_{m}\right)$. Let $d$ be the Krull dimension $\operatorname{dim} R$ of $R$, i.e., the maximum number of elements of $R$ which are algebraically independent over $k$. (Do not confuse the Krull dimension "dim" with the vector space dimension " $\operatorname{dim}_{k}$ ") Suppose $\theta_{1}, \ldots, \theta_{d}$ are homogeneous elements of $R$ of nonzero degree such that $\operatorname{dim}_{k} R /\left(\theta_{1}, \ldots, \theta_{d}\right)<$ $\infty$, or equivalently, such that $\operatorname{dim} R /\left(\theta_{1}, \ldots, \theta_{d}\right)=0$. Then $\theta_{1}, \ldots, \theta_{d}$ are called a homogeneous system of parameters (h.s.o.p.) for $R$. Again if we wish to emphasize that $\operatorname{deg} \theta_{i} \in \mathbf{N}^{m}, 1 \leqslant i \leqslant d$, then we call $\theta_{1}, \ldots, \theta_{d}$ an $\mathbf{N}^{m}$ homogeneous system of parameters. If $m=1$ then the Noether normalization lemma guarantees the existence of an h.s.o.p. Moreover, if $k$ is infinite and $R$ is generated by $R_{1}$, then we can choose each $\theta_{i}, 1 \leqslant i \leqslant d$, to have degree one. However, when $m>1$ an h.s.o.p. usually will not exist; indeed, a crucial point of this paper concerns the existence of an h.s.o.p. in certain situations when $m>1$ (Theorem 4.1). At any rate, suppose $\theta_{1}, \ldots, \theta_{d}$ is an h.s.o.p. for $R$. Let $S=R /\left(\theta_{1}, \ldots, \theta_{d}\right)$. Since $\theta_{1}, \ldots, \theta_{d}$ are homogeneous, $S$ inherits from $R$ the structure of an $\mathbf{N}^{m}$-graded $k$-algebra. We now say that $R$ is Cohen-Macaulay if

$$
F(R, \lambda)=F(S, \lambda) \prod_{i=1}^{d}\left(1-\lambda^{\operatorname{deg} \theta_{i}}\right)^{-1} .
$$

This is not the usual definition of a Cohen-Macaulay ring, but it is equivalent. For a reconciliation with the usual definition in terms of $R$-sequences, see [24]. It is important to realize that the question of whether or not $R$ is Cohen-Macaulay is independent of the grading chosen for $R$, though this is not immediately obvious from (1). Thus once we know that $R$ is CohenMacaulay, we know that (1) holds for whatever grading we choose for $R$. From the combinatorial point of view, the importance of Cohen-Macaulay rings $R$ is that the much smaller ring $S$ carries a lot of combinatorial information about $R$, in particular, the Hilbert function of $R$.

Now given a complex $\Delta$ on $V=\left\{x_{1}, \ldots, x_{n}\right\}$, associate with it a certain $\mathrm{N}$-graded $k$-algebra $A_{\Delta}$ as follows. Let $A=k\left[x_{1}, \ldots, x_{n}\right]$, the polynomial ring over $k$ on the vertices of $\Delta$. Let $I_{\Delta}$ be the ideal generated by all monomials $x_{i_{1}} x_{i_{2}} \cdots x_{i_{s}}$ with $i_{1}<i_{2}<\cdots<i_{s}$ and $\left\{x_{i_{1}}, x_{i_{2}}, \ldots, x_{i_{s}}\right\} \notin \Delta$. Define a grading on $A_{\Delta}=A / I_{\Delta}$ by setting $\operatorname{deg} x_{i}=1$. We say that $\Delta$ is a 
Cohen-Macaulay complex (always with respect to the field $k$ ) if $A_{\Delta}$ is a Cohen-Macaulay ring. This is the algebraic definition of a Cohen-Macaulay complex.

To give the topological definition, recall that if $F \in \Delta$, then the link of $F$ is the complex lk $F=\{G \in \Delta: F \cap G=\varnothing, F \cup G \in \Delta\}$. In particular, lk $\varnothing=$ $\Delta$.

1.2 Theorem. Let $\Delta$ be $a$-complex and $k$ a field. The following three conditions are equivalent.

(i) $\Delta$ is Cohen-Macaulay (over $k$ ).

(ii) For all $F \in \Delta, \tilde{H}_{i}(\mathrm{lk} F)=0$ if $i<\operatorname{dim}(\mathrm{lk} F)$. (Here $\tilde{H}$ denotes reduced simplicial homology with coefficient field $k$.)

(iii) Let $X=|\Delta|$, the geometric realization of $\Delta$, so that $\Delta$ is a triangulation of $X$. Then $\tilde{H}_{i}(X)=H_{i}(X, X-p)=0$ for all $i<\delta$ and all $p \in X$. (Here $\tilde{H}$ denotes reduced singular homology and $H$ relative singular homology, both over k.)

The equivalence of (i) and (ii) above is a theorem of G. Reisner [16], while the equivalence of (ii) and (iii) is a purely topological result first explicitly proved by J. Munkres [15, Theorem 2.1]. A stronger result was later proved by Hochster [10, Theorem 4.1].

Let us remark that the following results are immediate consequences of Theorem 1.2: (a) every Cohen-Macaulay complex is pure, (b) a CohenMacaulay complex of dimension greater than zero is connected, and (c) a graph ( = complex of dimension zero or one) is Cohen-Macaulay if and only if it has no edges or is connected.

Let $H(\Delta, m), m \in \mathbf{N}$, denote the Hilbert function of $A_{\Delta}$. It is easy to see [22, Proposition 3.2] that

$$
H(\Delta, m)= \begin{cases}1, & \text { if } m=0, \\
\sum_{i=0}^{\delta} f_{i}\left(\begin{array}{c}
m-1 \\
i
\end{array}\right), & \text { if } m>0,\end{cases}
$$

where $\left(f_{0}, f_{1}, \ldots, f_{\delta}\right)$ is the $f$-vector of $\Delta$. An immediate consequence of (2) (using [1, Theorem 11.4]) is the result $\operatorname{dim} A_{\Delta}=1+\operatorname{dim} \Delta=1+\delta$. Since $H(\Delta, m)$ is a polynomial in $m$ for $m \geqslant 1$, it follows that there are integers $1=h_{0}, h_{1}, \ldots, h_{\delta+1}$ such that

$$
(1-\lambda)^{1+\delta} F\left(A_{\Delta}, \lambda\right)=h_{0}+h_{1} \lambda+\cdots+h_{1+\delta} \lambda^{1+\delta} .
$$

The vector $\mathbf{h}=\mathbf{h}(\Delta)=\left(h_{0}, h_{1}, \ldots, h_{1+\delta}\right)$ is called the $h$-vector of $\Delta$. We wish to state a characterization analogous to Theorem 1.1 of the $h$-vector of a Cohen-Macaulay complex $\Delta$. To do so, recall that a multiset $M$ on a set $S$ is a set with repeated elements belonging to $S$. More precisely, $M$ is a function $S \rightarrow \mathrm{N}$, where $M(x)$ is regarded as the number of repetitions of $x \in S$. The 
cardinality of $M$ is card $M=\Sigma_{x \in S} M(x)$. A multiset $M^{\prime}: S \rightarrow \mathbf{N}$ is a submultiset of $M$ (denoted $M^{\prime} \subset M$ ) if $M^{\prime}(x) \leqslant M(x)$ for all $x \in S$. A multicomplex is a collection of multisets such that if $M \in \Lambda$ and $M^{\prime} \subset M$, then $M^{\prime} \in \Lambda$. The dimension and $f$-vector of a multicomplex are defined in the obvious way in analogy with complexes, i.e., $f_{i}=\operatorname{card}\{M \in \Lambda$ : $\operatorname{card} M$ $=i+1\}$ and $\operatorname{dim} \Lambda=\max \left\{i: f_{i} \neq 0\right\}$. Any vector $\left(f_{0}, f_{1}, \ldots, f_{\delta}\right)$ which is the $f$-vector of a multicomplex is called an $M$-vector. We allow $f_{j+1}=f_{j+2}$ $=\cdots=f_{\delta}=0$ in an $M$-vector; if also $f_{j} \neq 0$ this means that the corresponding multicomplex $\Lambda$ has dimension $j$.

In analogy with Theorem 1.1 we have the following result essentially due to Macaulay [13] (explaining our terminology " $M$-vector"). A common generalization of Theorems 1.1 and 1.3 appears in [3], and an exposition of these results appears in [9].

1.3 THEOREM. Given positive integers $f$ and $i$, write

$$
f=\left(\begin{array}{c}
n_{i} \\
i
\end{array}\right)+\left(\begin{array}{c}
n_{i-1} \\
i-1
\end{array}\right)+\cdots+\left(\begin{array}{c}
n_{j} \\
j
\end{array}\right)
$$

where $n_{i}>n_{i-1}>\cdots>n_{j} \geqslant j \geqslant 1$ (exactly as in Theorem 1.1); and define

$$
f^{\langle i\rangle}=\left(\begin{array}{c}
n_{i}+1 \\
i+1
\end{array}\right)+\cdots+\left(\begin{array}{c}
n_{j}+1 \\
j+1
\end{array}\right),
$$

with $0^{\langle i\rangle}=0$. Then the vector $\mathbf{f}=\left(f_{0}, f_{1}, \ldots, f_{\delta}\right)$ of nonnegative integers is an $M$-vector if and only if $f_{i+1} \leqslant f_{i}^{\langle i+1\rangle}$ for $0 \leqslant i \leqslant \delta-1$.

We can now give the characterization [23, Theorem 6] of the $h$-vector of a Cohen-Macaulay complex.

1.4 Theorem. A vector $\left(h_{0}, h_{1}, \ldots, h_{\delta+1}\right)$ is the $h$-vector of a CohenMacaulay complex of dimension $\delta$ if and only if $h_{0}=1$ and $\left(h_{1}, h_{2}, \ldots, h_{\delta+1}\right)$ is an $M$-vector.

2. Balanced complexes. We wish to introduce a class of Cohen-Macaulay complexes for which Theorem 1.4 can be considerably strengthened and refined. Recall that an ordered partition of a finite set $V$ is a sequence $\left(V_{1}, \ldots, V_{m}\right)$ of nonvoid, pairwise disjoint subsets of $V$ satisfying $V_{1}$ $\cup \cdots \cup V_{m}=V$.

Definition. A balanced complex of type $\left(a_{1}, \ldots, a_{m}\right)$ is a pair $(\Delta, \pi)$ satisfying:

(i) $\Delta$ is a pure $\delta$-complex on a vertex set $V$,

(ii) $\pi=\left(V_{1}, \ldots, V_{m}\right)$ is an ordered partition of $V$, and

(iii) for every maximal face $F \in \Delta$ and every $i \in[m]$, we have card $(F \cap$ $\left.V_{i}\right)=a_{i}$. (Hence $a_{1}+\cdots+a_{m}=\delta+1$.) 
A balanced complex of type $(1,1, \ldots, 1)$ is called completely balanced. Note that a balanced complex of type $(\delta+1)$ (i.e., with $m=1)$ is really nothing more than a pure $\delta$-complex, since condition (iii) holds automatically. We could have altered our definition somewhat so that $\Delta$ need not be pure, but nothing significant is gained by doing so. In particular, we are primarily concerned with Cohen-Macaulay complexes, and these are always pure.

We now give some examples of completely balanced complexes. Let $P$ be a poset (= partially ordered set) on a finite set $V$, and define $\Delta(P)$ to be the complex on $V$ whose faces are the chains (= linearly ordered subsets) of $P$. We will use such terminology as " $P$ is pure" or " $P$ is Cohen-Macaulay" to mean the corresponding statement for $\Delta(P)$. Thus $P$ is pure if and only if all maximal chains of $P$ have the same length, and $\operatorname{dim} P$ is the length of the longest chain in $P$. Moreover, a Cohen-Macaulay poset $P$ is one for which $\Delta(P)$ is a Cohen-Macaulay complex. Suppose now that $P$ is pure of dimension $\delta$. If $x \in V$, let $\rho(x)$ be the largest integer $r$ for which there is a chain $x_{1}<x_{2}<\cdots<x_{r}=x$ in $P$. We call $\rho(x)$ the rank of $x$ and $\rho$ the rank function of $P$. (Some authors would call $\rho(x)-1$ the rank or height of $x$.) If we set $V_{i}=\{x \in P: \rho(x)=i\}, 1 \leqslant i \leqslant \delta+1$, then clearly $\pi=$ $\left(V_{1}, V_{2}, \ldots, V_{\delta+1}\right)$ is an ordered partition of $V$ and $(\Delta(P), \pi)$ is completely balanced. We call $\pi$ the standard ordered partition of $P$. If $\Delta$ is any complex, let $Q=Q(\Delta)$ be the poset of nonvoid faces of $\Delta$, ordered by inclusion. Then $\Delta(Q)$ is just the first barycentric subdivision of $\Delta$. Hence any space $X$ which possesses a finite pure triangulation possesses a completely balanced triangulation. There does not seem to be a nice characterization of completely balanced complexes, though a sufficient condition for $\Delta$ to be completely balanced is mentioned in [5]. On the other hand, one can characterize complexes $\Delta$ of the form $\Delta(P)$ for a finite poset $P$. Namely, it is necessary and sufficient that $\Delta$ satisfy the following:

(i) any minimal set of vertices which do not form a face of $\Delta$ has two elements (i.e., the ideal $I_{\Delta}$ is generated by quadratic monomials), and

(ii) let $\Gamma$ be the 1-skelton of $\Delta$. Then $\Gamma$ must satisfy the well-known conditions of Gilmore and Hoffman [8], Ghouilà-Houri [7], or Gallai [6] for being a comparability graph.

3. Numerical invariants of balanced complexes. If $(\Delta, \pi)$ is a balanced complex of type $\mathbf{a}=\left(a_{1}, a_{2}, \ldots, a_{m}\right)$ and if $\mathbf{b}=\left(b_{1}, b_{2}, \ldots, b_{m}\right) \in \mathbf{N}^{m}$, then define $f_{\mathbf{b}}=f_{\mathbf{b}}(\Delta, \pi)$ to be the number of faces $F \in \Delta$ for which $\operatorname{card}\left(F \cap V_{i}\right)$ $=b_{i}, 1 \leqslant i \leqslant m$. Note that $f_{\mathbf{b}}=0$ unless $b_{i} \leqslant a_{i}$ for all $i$ (written $\mathbf{b} \leqslant \mathbf{a}$ ). Note also that $f_{i}(\Delta)=\sum f_{\mathbf{b}}(\Delta, \pi)$, where the sum is over all vectors $\mathbf{b} \leqslant \mathbf{a}$ such that $\sum b_{j}=i+1$. Hence the numbers $f_{\mathbf{b}}$ are a refinement of the numbers $f_{i}$. 
If $S \subset[m]$, let $\left(\Delta_{S}, \pi_{S}\right)$ be the balanced complex defined as follows:

(i) $\Delta_{S}=\left\{F \in \Delta: F \cap V_{i}=\varnothing\right.$ if $\left.i \notin S\right\}$,

(ii) if

$$
S=\left\{c_{1}, c_{2}, \ldots, c_{r}\right\} \text { with } c_{1}<c_{2}<\cdots<c_{r},
$$

then $\pi_{S}=\left(V_{c_{1}}, V_{c_{2}}, \ldots, V_{c_{r}}\right)$.

Hence $\left(\Delta_{S}, \pi_{S}\right)$ is balanced of type $\left(a_{c_{1}}, a_{c_{2}}, \ldots, a_{c_{r}}\right)$.

For instance, if $P$ is a pure poset of dimension $\delta$ and if $\pi$ is the standard ordered partition of $P$, then $\Delta(P)_{S}=\Delta\left(P_{S}\right)$, where $P_{S}$ is the poset obtained from $P$ by removing all elements whose ranks do not belong to $S$. In particular, $P_{[\gamma]}$ is the so-called "rank $\gamma$ upper-truncation of $P$ ". Note that in general if $(\Delta, \pi)$ has type $\left(a_{1}, \ldots, a_{m}\right)$, then $\left(\Delta_{[m]}, \pi_{[m]}\right)=(\Delta, \pi)$, and $\Delta_{\varnothing}=\varnothing$.

The following result is an immediate consequence of the definition of $\left(\Delta_{S}, \pi_{S}\right)$.

3.1 Proposition. Let $(\Delta, \pi)$ be a balanced complex of type $\left(a_{1}, a_{2}, \ldots, a_{m}\right)$ $=$ a. Let $S \subset[\mathrm{m}]$, say $S=\left\{c_{1}, c_{2}, \ldots, c_{r}\right\}$ with $c_{1}<c_{2}<\cdots<c_{r}$, so that $\left(\Delta_{S}, \pi_{S}\right)$ is balanced of type $\left(a_{c_{1}}, a_{c_{2}}, \ldots, a_{c_{r}}\right)=\mathbf{a}^{\prime}$. If $\left(b_{c_{1}}, \ldots, b_{c_{r}}\right)=\mathbf{b}^{\prime} \leqslant \mathbf{a}^{\prime}$, define $\left(b_{1}, \ldots, b_{m}\right)=\mathbf{b}$ by letting $b_{i}=0$ if $i$ is not one of the $c_{j}$. Then $f_{\mathbf{b}^{\prime}}\left(\Delta_{S}, \pi_{S}\right)=f_{\mathbf{b}}(\Delta, \pi)$.

The significance of Proposition 3.1 is the following. If we know the numbers $f_{\mathbf{b}}(\Delta, \pi)$ for all $\mathbf{b}$, then we also know the numbers $f_{\mathbf{b}^{\prime}}\left(\Delta_{S}, \pi_{S}\right)$ for all $S \subset[m]$ and all $\mathbf{b}^{\prime}$.

We can refine the $h$-vector of a balanced $\delta$-complex $(\Delta, \pi)$, where $\pi=$ $\left(V_{1}, \ldots, V_{m}\right)$, just as we did the $f$-vector, as follows. We make the ring $A_{\Delta}$ into an $\mathbf{N}^{m}$-graded $k$-algebra by defining, for a vertex $x$ of $\Delta, \operatorname{deg} x$ to be the $i$ th unit coordinate vector $\mathbf{e}_{i} \in \mathbf{N}^{m}$. Equivalently, $\lambda^{\operatorname{deg} x}=\lambda_{i}$ if $x \in V_{i}$.

3.2 Proposition. Let $(\Delta, \pi)$ be a balanced complex of type $\mathbf{a}=\left(a_{1}, \ldots, a_{m}\right)$, where $\pi=\left(V_{1}, \ldots, V_{m}\right)$, and let $H\left(A_{\Delta}, \mathbf{b}\right)$ denote the Hilbert function of $A_{\Delta}$ with the above $\mathbf{N}^{m}$-grading. Then for all $\mathbf{b}=\left(b_{1}, \ldots, b_{m}\right) \in \mathbf{N}^{m}$,

$$
H\left(A_{\Delta}, \mathbf{b}\right)=\sum_{\mathbf{c}} f_{\mathbf{c}}(\Delta, \pi) \prod_{b_{i}>0}\left(\begin{array}{l}
b_{i}-1 \\
c_{i}-1
\end{array}\right),
$$

where the sum is over all $\left(c_{1}, \ldots, c_{m}\right)=\mathbf{c} \leqslant \mathbf{a}$ such that $c_{i}=0 \Leftrightarrow b_{i}=0$.

Note that Proposition 3.2 reduces to (2) when $m=1$.

Proof of Proposition 3.2. If $M=x_{1}^{\alpha_{1}} \cdots x_{n}^{\alpha_{n}}$ is a nonzero monomial appearing in $A_{\Delta}$, then define the support of $M$ by $\operatorname{supp} M=\left\{x_{i} \in V\right.$ : $\left.\alpha_{i}>0\right\}$. Note supp $M \in \Delta$. Given $F \in \Delta$ with $c_{i}=\operatorname{card} F \cap V_{i}$, the number of monomials $M$ satisfying $\operatorname{supp} M=F$ and $\operatorname{deg} M=\mathbf{b}$ is $\Pi_{i: b_{i}>0}\left(\begin{array}{l}b_{i}-1 \\ c_{i}-1\end{array}\right)$, since there are $\left(\begin{array}{c}b_{i}-1 \\ c_{i}-1\end{array}\right)$ monomials of degree $b_{i}$ in $c_{i}$ variables with each variable having positive exponent. Summing over all $F \in \Delta$ completes the proof. 
It is an immediate consequence of Proposition 3.2 , or can be easily seen directly, that

$$
F\left(A_{\Delta}, \lambda\right)=\sum_{F \in \Delta} \prod_{x \in F} \lambda_{\rho(x)}\left(1-\lambda_{\rho(x)}\right)^{-1},
$$

where for each $x \in V, \rho(x)$ is defined by $x \in V_{\rho(x)}$.

Hence we obtain the following result, which will be of use later.

3.3 Proposition. Let $(\Delta, \pi)$ be a balanced complex of type $\left(a_{1}, \ldots, a_{m}\right)$. Then $F\left(A_{\Delta}, \lambda\right) \Pi_{i=1}^{m}\left(1-\lambda_{i}\right)^{a_{i}}$ (or equivalently $F\left(A_{\Delta}, \lambda\right) \Pi_{x \in F}\left(1-\lambda_{\rho(x)}\right)$ for any maximal face $F$ of $\Delta)$ is a polynomial $P\left(A_{\Delta}, \lambda\right)$ in $\lambda_{1}, \ldots, \lambda_{m}$. Moreover, the degree of $P\left(A_{\Delta}, \lambda\right)$ with respect to $\lambda_{i}$ is no more than $a_{i}$. In particular, if $(\Delta, \pi)$ is completely balanced then every monomial appearing in $P\left(A_{\Delta}, \lambda\right)$ is squarefree.

Now if $\mathbf{b} \in \mathbf{N}^{m}$ define $h_{\mathbf{b}}=h_{\mathbf{b}}(\Delta, \pi)$ to be the coefficient of $\lambda^{\mathbf{b}}$ in the polynomial $F\left(A_{\Delta}, \lambda\right) \prod_{i=1}^{m}\left(1-\lambda_{i}\right)^{a_{i}}$. Proposition 3.3 asserts that

$$
h_{\mathbf{b}}=0 \text { unless } \mathbf{b} \leqslant \mathbf{a} \text {. }
$$

Clearly $h_{i}(\Delta)=\Sigma h_{\mathbf{b}}(\Delta, \pi)$, where b ranges over all $\left(b_{1}, \ldots, b_{m}\right) \in \mathbf{N}^{m}$ satisfying $b_{1}+\cdots+b_{m}=i$. Hence the numbers $h_{\mathrm{b}}$ are a refinement of the numbers $h_{i}$. Note that

$$
\begin{aligned}
F\left(A_{\Delta}, \lambda\right) \prod_{i=1}^{n}\left(1-\lambda_{i}\right)^{a_{i}} & =\left[\sum_{F \in \Delta} \prod_{x \in F} \lambda_{\rho(x)}\left(1-\lambda_{\rho(x)}\right)^{-1}\right] \prod_{i=1}^{n}\left(1-\lambda_{i}\right)^{a_{i}} \\
& =\sum_{\mathbf{c}} \sum_{\substack{F \in \Delta \\
\operatorname{card}\left(F \cap V_{i}\right)=c_{i}}} \lambda^{\mathbf{c}} \prod_{i=1}^{n}\left(1-\lambda_{i}\right)^{a_{i}-c_{i}} \\
& =\sum_{\mathbf{c}} f_{\mathbf{c}}(\Delta, \pi) \lambda^{\mathbf{c}} \prod_{i=1}^{n}\left(1-\lambda_{i}\right)^{a_{i}-c_{i}}
\end{aligned}
$$

from which we get

$$
h_{\mathbf{b}}(\Delta, \pi)=\sum_{\mathbf{c}<\mathbf{b}} f_{\mathbf{c}}(\Delta, \pi) \prod_{i=1}^{m}(-1)^{b_{i}-c_{i}}\left(\begin{array}{l}
a_{i}-c_{i} \\
b_{i}-c_{i}
\end{array}\right) .
$$

3.4 ExAmple. (a) Let $\Delta=\langle a b c, a b e, a c d, a d e, b c g$, bef, bfg, cdg, def, dfg $\rangle$, so $|\Delta| \approx \mathbf{S}^{2}$. Let $V_{1}=\{a, c, e, f, g\}, V_{2}=\{b, d\}$ and $\pi=\left(V_{1}, V_{2}\right)$. Then $(\Delta, \pi)$ is balanced of type $(2,1)$. We have (writing $f_{r s}$ for $\left.f_{(r, s)}\right) f_{00}=1, f_{10}=5$, $f_{01}=2, f_{20}=5, f_{11}=10, f_{21}=10$. Also (writing $\lambda=(\mu, \lambda)$ ),

$$
\begin{aligned}
F\left(A_{\Delta}, \lambda\right)= & (1-\mu)^{2}(1-\lambda)+5 \mu(1-\mu)(1-\lambda)+2 \lambda(1-\mu)^{2} \\
& +5 \mu^{2}(1-\lambda)+10 \mu \lambda(1-\mu)+10 \mu^{2} \lambda \\
= & 1+3 \mu+\lambda+\mu^{2}+3 \mu \lambda+\mu^{2} \lambda .
\end{aligned}
$$


Hence $h_{00}=h_{01}=h_{20}=h_{21}=1, h_{10}=h_{11}=3$.

(b) Suppose $\Delta=\langle a b, c d\rangle$ with $V_{1}=\{a, c\}, V_{2}=\{b, d\}$, and $\pi=$ $\left(V_{1}, V_{2}\right)$. Then $(\Delta, \pi)$ is of type $(1,1)$, i.e., is completely balanced. We have $f_{00}=1, f_{10}=f_{01}=f_{11}=2$. Also

$$
\begin{aligned}
F\left(A_{\Delta}, \lambda\right) & =(1-\mu)(1-\lambda)+2 \lambda(1-\mu)+2 \mu(1-\lambda)+2 \mu \lambda \\
& =1+\mu+\lambda-\mu \lambda .
\end{aligned}
$$

Hence $h_{00}=h_{10}=h_{01}=1, h_{11}=-1$.

In general it is difficult to obtain any intuition for the numbers $h_{\mathrm{b}}(\Delta, \pi)$. There are two special circumstances, however, in which they have additional interpretations. First define the reduced Euler characteristic $\tilde{\chi}(\Delta)$ of a complex $\Delta$ by $\tilde{\chi}(\Delta)=\chi(\Delta)-1$, where $\chi(\Delta)$ is the usual Euler characteristic. Equivalently, $\tilde{\chi}(\Delta)=-1+f_{0}(\Delta)-f_{1}(\Delta)+\ldots$ In particular, $\tilde{\chi}(\varnothing)=-1$. Note that if the reduced homology of $\Delta$ satisfies $\tilde{H}_{i}(\Delta)=0$ for $i<\delta=\operatorname{dim} \Delta$, then $(-1)^{\delta} \tilde{\chi}(\Delta)=\operatorname{dim}_{k} \tilde{H}_{\delta}(\Delta) \geqslant 0$.

3.5 Proposition. Let $(\Delta, \pi)$ be a balanced complex of type $\left(a_{1}, \ldots, a_{m}\right)$. Let $S \subset[m]$, and define $\mathbf{b}=\left(b_{1}, \ldots, b_{m}\right)$ by

$$
b_{i}= \begin{cases}a_{i}, & \text { if } i \in S, \\ 0, & \text { if } i \notin S .\end{cases}
$$

Let $\left(\Delta_{S}, \pi_{S}\right)$ be the balanced complex defined by (3). Then $h_{\mathrm{b}}(\Delta, \pi)=$ $(-1)^{\delta} \tilde{\chi}\left(\Delta_{S}\right)$, where $\delta=b_{1}+\cdots+b_{m}-1=\operatorname{dim} \Delta_{S}$.

Proof. Let $\mathbf{c}=\left(c_{1}, \ldots, c_{m}\right) \leqslant \mathbf{b}$. For all $i$ we have $\left(\begin{array}{c}a_{i}-c_{i} \\ b_{i}-c_{i}\end{array}\right)=1$, since $b_{i}=a_{i}$ or $b_{i}=c_{i}=0$. Hence from (6),

$$
\begin{aligned}
h_{\mathbf{b}}(\Delta, \pi) & =\sum_{\mathbf{c}<\mathbf{b}} f_{\mathbf{c}}(\Delta, \pi) \prod_{i=1}^{m}(-1)^{b_{i}-c_{i}} \\
& =(-1)^{\delta} \sum_{i=-1}^{\delta} f_{i}\left(\Delta_{S}\right)(-1)^{i} \quad\left(\text { with } f_{-1}(\Delta)=1\right) \\
& =(-1)^{\delta} \tilde{\chi}\left(\Delta_{S}\right) .
\end{aligned}
$$

Note that if $(\Delta, \pi)$ is completely balanced then any $\mathbf{b} \leqslant \mathbf{a}$ satisfies the hypothesis of Proposition 3.5. Hence in the completely balanced case every $h_{\mathrm{b}}(\Delta, \pi)$ may be interepreted as a reduced Euler characteristic (up to sign).

For our second alternative interpretation of the numbers $h_{\mathrm{b}}(\Delta, \pi)$, we need to define the notion of "shellability." Our definition is slightly more general than that sometimes given, e.g., [4]. If $\Delta$ is a pure $\delta$-complex, then a shelling of $\Delta$ is an ordering $F_{1}, F_{2}, \ldots, F_{r}$ of the $\delta$-faces of $\Delta$ (so $r=f_{\delta}(\Delta)$ ) such that for $1 \leqslant i \leqslant r-1,\left(F_{1} \cup F_{2} \cup \cdots \cup F_{i}\right) \cap F_{i+1}$ is a nonvoid union of $(\delta-1)$ faces of $F_{i+1}$. In exactly the same manner as McMullen's interpretation [14, p. 
182] of $h_{i}(\Delta)$ when $\Delta$ is shellable (McMullen uses $g_{i-1}^{(d)}$ for our $h_{i}$ ), we obtain the following result.

3.6 Proposition. Let $(\Delta, \pi)$ be a balanced complex where $\pi=$ $\left(V_{1}, \ldots, V_{m}\right)$, and let $F_{1}, F_{2}, \ldots, F_{r}$ be a shelling of $\Delta$. For each $i \in[r]$, define $G_{i}$ to be the unique minimal face of $F_{i}$ which is not contained in $F_{1} \cup F_{2}$ $\cup \cdots \cup F_{i-1}$. (In particular, $G_{1}=\varnothing$.) Define $\mathbf{b}(i)=\left(b_{1}(i), \ldots, b_{m}(i)\right)$ by $b_{j}(i)=$ card $G_{i} \cap V_{j}$. Then $h_{\mathbf{b}}(\Delta, \pi)$ is equal to the number of integers $i \in[m]$ for which $\mathbf{b}=\mathbf{b}(i)$.

Proof. Let $F_{i}(\lambda)$ be the Poincare series for the pure subcomplex of $\Delta$ whose maximal faces are $F_{1}, F_{2}, \ldots, F_{i}$. Then by the definition of $G_{i}$ and $\mathbf{b}(i)$,

$$
F_{i+1}(\lambda)=F_{i}(\lambda)+\frac{\lambda^{\mathbf{b}(i)}}{\prod_{i=1}^{m}\left(1-\lambda_{i}\right)^{a_{i}}},
$$

so that

$$
F\left(A_{\Delta}, \lambda\right) \prod_{i=1}^{m}\left(1-\lambda_{i}\right)^{a_{i}}=\sum_{i=1}^{m} \lambda^{b(i)} .
$$

The proof now follows from the definition of $h_{\mathrm{b}}(\Delta, \pi)$.

3.7 EXAMPLE. Let $(\Delta, \pi)$ be the balanced complex of Example 3.4(a). Then (writing $a b c$ for $\{a, b, c\}$, etc.) abc, acd, ade, abe, cdg, dfg, def, bef, bfg, bcg is a shelling of $\Delta$. We have $G_{1}=\varnothing, G_{2}=d, G_{3}=e, G_{4}=b e, G_{5}=g, G_{6}=f$, $G_{7}=e f, G_{8}=b f, G_{9}=b g, G_{10}=b c g$. Since $V_{1}=\{a, c, e, f, g\}$ and $V_{2}=$ $\{b, d\}$, we have $\mathbf{b}(1)=(0,0), \mathbf{b}(2)=(0,1), \mathbf{b}(3)=(1,0), \mathbf{b}(4)=(1,1), \mathbf{b}(5)=$ $(1,0), \mathbf{b}(6)=(1,0), \mathbf{b}(7)=(2,0), \mathbf{b}(8)=(1,1), \mathbf{b}(9)=(1,1), \mathbf{b}(10)=(2,1)$, and we obtain the same values for $h_{\mathrm{b}}(\Delta, \pi)$ as before.

Proposition 3.6 shows that $h_{\mathrm{b}}(\Delta, \pi) \geqslant 0$ when $\Delta$ is shellable. However, it is easy to see that all shellable complexes are Cohen-Macaulay, so that the inequality $h_{\mathrm{b}}(\Delta, \pi) \geqslant 0$ is subsumed and generalized by Theorem 4.4 below. For a survey of some aspects of the subject of shellability, see [4]. Examples of shellable complexes include: (i) the boundary complex of a simplicial convex polytope (but not necessarily a triangulation of a sphere), (ii) connected graphs and triangulations of 2-cells, (iii) the independent set complex and broken circuit complex [23, §7] of a finite matroid, and (iv) the complex $\Delta(P)$ where $P$ is an admissible lattice in the sense of [21]. Example (iii) is due to Scott Provan and (iv) to Anders Bjørner.

There is a somewhat weaker condition than shellability which implies that $h_{\mathrm{b}}(\Delta, \pi) \geqslant 0$. If $\Delta$ is a complex and if $G \subset F$ are faces of $\Delta$, then define the interval $[G, F]$ by $[G, F]=\left\{F^{\prime} \mid G \subset F^{\prime} \subset F\right\}$. An upper partition of a pure $\delta$-complex $\Delta$ is a collection $\left[G_{1}, F_{1}\right], \ldots,\left[G_{r}, F_{r}\right]$ of intervals of $\Delta$ satisfying:

(i) $\left[G_{i}, F_{i}\right] \cap\left[G_{j}, F_{j}\right]=\varnothing$ if $i \neq j$,

(ii) $\Delta=\left[G_{1}, F_{1}\right] \cup \cdots \cup\left[G_{r}, F_{r}\right]$, 
(iii) $\operatorname{dim} F_{i}=\delta$ for all $i \in[r]$ (so $r=f_{\delta}(\Delta)$ ).

A complex $\Delta$ possessing an upper partiton is said to be partitionable. It is easily seen that every shellable complex is partitionable. The converse is false. In fact, if $\Delta=\langle a b, c d, c e, d e\rangle$ then $\Delta$ is partitionable but not even CohenMacaulay. An upper partition of $\Delta$ is given by [ $\varnothing, a b],[c, c d],[d, d e],[e, c e]$. We do not know if every Cohen-Macaulay complex is partitionable. Now assume $(\Delta, \pi)$ is balanced with $\pi=\left(V_{1}, \ldots, V_{m}\right)$, and suppose $\left[G_{1}, F_{1}\right], \ldots,\left[G_{r}, F_{r}\right]$ is an upper partition of $\Delta$. Then as a slight generalization of Proposition 3.6, it is easily shown that if $\mathbf{b}=\left(b_{1}, \ldots, b_{m}\right)$, then $h_{\mathrm{b}}(\Delta, \pi)$ is equal to the number of $j \in[r]$ for which card $G_{j} \cap V_{i}=b_{i}$ for all $i \in[m]$. Partitionable complexes were independently considered by Provan [25, Appendix 4].

4. Balanced Cohen-Macaulay complexes. A balanced complex $(\Delta, \pi)$ for which $\Delta$ is a Cohen-Macaulay complex is called a balanced Cohen-Macaulay complex. Our main aim is to give restrictions on the numbers $h_{\mathbf{b}}$ associated with a balanced Cohen-Macaulay complex which strengthens and refines Theorem 1.4. Our results are based on the following fundamental algebraic property of balanced complexes.

4.1 ThEOREM. Let $(\Delta, \pi)$ be a balanced complex of type $\left(a_{1}, \ldots, a_{m}\right)$, where $\pi=\left(V_{1}, \ldots, V_{m}\right)$. Let $\Delta_{i}$ stand for the complex $\Delta_{\{i\}}$ of (3), so that $\Delta_{i}$ is just the restriction of $\Delta$ to $V_{i}$. Set $A_{i}=A_{\Delta_{i}}$, and give this ring an $\mathrm{N}$-grading by defining $\operatorname{deg} x=1$ for $x \in V_{i}$. Now give $A_{\Delta}$ the $\mathbf{N}^{m}$-grading $\operatorname{deg} x=\mathbf{e}_{i}$ if $x \in V_{i}$ (as defined preceding Proposition 3.2). Suppose $\Psi_{i}$ is an $\mathbf{N}$-homogeneous system of parameters for $A_{i}$. Then $\Psi=\Psi_{1} \cup \cdots \cup \Psi_{m}$ is an $\mathbf{N}^{m}$-homogeneous system of parameters for $A_{\Delta}$.

Proof. If $\theta \in \Psi_{i}$ has degree $\alpha \in \mathbf{N}$ in $A_{i}$, then $\theta$ is $\mathbf{N}^{m}$-homogeneous in $A_{\Delta}$, with $\operatorname{deg} \theta=\alpha \mathbf{e}_{i} \in \mathbf{N}^{m}$. Since $a_{1}+a_{2}+\cdots+a_{m}=\operatorname{dim} A_{\Delta}$, it remains only to show $\operatorname{dim}_{k} A_{\Delta} /(\Psi)<\infty$, where $(\Psi)$ denotes the ideal generated by all $\theta \in \Psi$. Now $A_{\Delta}$ is a quotient ring of $A_{1} \otimes_{k} A_{2} \otimes_{k} \cdots \otimes_{k} A_{m}$, so

$$
\operatorname{dim}_{k} A_{\Delta} /(\Psi) \leqslant \prod_{i=1}^{m} \operatorname{dim}_{k} A_{i} /\left(\Psi_{i}\right)<\infty .
$$

This completes the proof.

4.2 Corollary. Let $(\Delta, \pi)$ be completely balanced with $\pi=\left(V_{1}, \ldots, V_{m}\right)$. Let $\theta_{i}=\Sigma_{x \in V_{i}} x$. Then $\theta_{1}, \ldots, \theta_{m}$ is an $\mathbf{N}^{m}$-homogeneous system of parameters for $A_{\Delta}$. Indeed, $\operatorname{deg} \theta_{i}=\mathbf{e}_{i}$.

Proof. We have $A_{i}=k\left[V_{i}\right] / I_{i}$, where $I_{i}$ is generated by all products $x x^{\prime}$ such that $x, x^{\prime} \in V_{i}$ and $x \neq x^{\prime}$. From this it follows that the single element 
$\theta_{i}$ is a system of parameters for $A_{i}$, and the proof follows from Theorem 4.1.

Remark. When $(\Delta, \pi)$ is completely balanced, Corollary 4.2 gives an h.s.o.p. for $A_{\Delta}$ consisting of linear forms (i.e., of $\mathbf{N}$-degree one). There is a more general result which gives a necessary and sufficient condition for a set of linear forms to be an N-h.s.o.p. for any $A_{\Delta}$. Let $d=\operatorname{dim} A_{\Delta}=1+\operatorname{dim} \Delta$, and let

$$
\theta_{i}=\sum_{j=1}^{n} \alpha_{i j} x_{j}, \quad \alpha_{i j} \in k, 1 \leqslant i \leqslant d,
$$

be a set of $d$ linear forms in $A_{\Delta}$. Then $\theta_{1}, \ldots, \theta_{d}$ is an $\mathbf{N}$-h.s.o.p. if and only if for every $F \in \Delta$ (equivalently, for every maximal $F \in \Delta$ ) the $d \times($ card $F$ ) matrix $\left(\alpha_{i j}\right)$, where $1 \leqslant i \leqslant d$ and $x_{j} \in F$, has rank equal to card $F$. We omit the proof.

Theorem 4.1 allows an easy proof of the next result.

4.3 Theorem. Let $(\Delta, \pi)$ be a balanced Cohen-Macaulay complex of type $\left(a_{1}, \ldots, a_{m}\right)$, and let $S \subset[m]$. Then $\left(\Delta_{S}, \pi_{S}\right)$ is a balanced Cohen-Macaulay complex of type $\left(a_{c_{1}}, a_{c_{2}}, \ldots, a_{c_{r}}\right)$, where $S=\left\{c_{1}, c_{2}, \ldots, c_{r}\right\}$ and $c_{1}<c_{2}$ $<\cdots<c_{r}$.

Proof. We only need to prove that $\Delta_{S}$ is Cohen-Macaulay. By Theorem 1.2 , the desired result is of a purely topological nature. An almost equivalent result was first proved by J. Munkres [15, Theorem 6.4] using topological methods, and his proof straightforwardly extends to Theorem 4.3. However, it may be of interest to give a simple alternative proof based directly on the definition (1) of a Cohen-Macaulay ring.

Let $A_{\Delta}$ have the usual $\mathrm{N}^{m}$-grading defined by $\operatorname{deg} x=\mathbf{e}_{i}$ if $x \in V_{i}$. Let $\Psi=\psi_{1} \cup \cdots \cup \Psi_{m}$ be a homogeneous system of parameters of the type described by Theorem 4.1. Then by (1),

$$
F\left(A_{\Delta}, \lambda\right) \prod_{\theta \in \Psi}\left(1-\lambda^{\operatorname{deg} \theta}\right)=F\left(A_{\Delta} /(\Psi), \lambda\right) .
$$

Since $\Delta_{S}$ consists of those faces $F \in \Delta$ for which $x \in V_{c_{1}} \cup \cdots \cup V_{c_{r}}$ whenever $x \in F$, it follows that $F\left(A_{\Delta_{s}}, \lambda\right)$ is obtained from $F\left(A_{\Delta}, \lambda\right)$ by setting $\lambda_{i}=0$ if $i \notin S$ and then substituting $\lambda_{i}$ for $\lambda_{c_{i}}$. Let $\Psi_{S}=\cup_{i \in S} \Psi_{i}$. By Theorem 4.1, $\Psi_{S}$ is an h.s.o.p. for $A_{\Delta_{s}}$. If $\operatorname{deg}\left(\right.$ resp. $\operatorname{deg}_{S}$ ) denotes degree in $A_{\Delta}$ (resp. $\left.A_{\Delta_{s}}\right)$, it follows that $\prod_{\theta \in \Psi_{s}}\left(1-\lambda^{\operatorname{deg}_{s} \theta}\right)$ is obtained from $\prod_{\theta \in \Psi}(1-$ $\left.\lambda^{\operatorname{deg} \theta}\right)$ by the same substitution as above. Now note that $A_{\Delta_{S}} /\left(\Psi_{S}\right)=$ $A_{\Delta} /(\Psi, X)$, where $X$ consists of all $x \in V$ such that $x \notin V_{i}$. for any $i \in S$. Since $A_{\Delta} /(\Psi)$ is $\mathrm{N}^{m}$-graded, a $k$-basis for $A_{\Delta} /(\Psi, X)$ consists of those monomials in $A_{\Delta} /(\Psi)$ whose support lies in $V_{c_{1}} \cup \cdots \cup V_{c_{r}}$. The remaining monomials in $A_{\Delta} /(\Psi)$ are zero modulo $X$. Hence $F\left(A_{\Delta} /(\Psi, X), \lambda\right)$ is 
obtained from $F\left(A_{\Delta} /(\Psi), \lambda\right)$ by making once again the same substitution $\lambda_{i} \rightarrow 0$ if $i \notin S$ and then $\lambda_{c_{i}} \rightarrow \lambda_{i}$. Hence when we make this substitution in (7) we obtain

$$
F\left(A_{\Delta_{s}}, \lambda\right) \prod_{\theta \in \Psi_{s}}\left(1-\lambda^{\operatorname{deg}_{s} \theta}\right)=F\left(A_{\Delta_{s}} /\left(\Psi_{S}\right), \lambda\right) .
$$

By (1), it follows that $A_{\Delta_{s}}$ is Cohen-Macaulay.

We are now in a position to discuss the $h$-vectors of balanced CohenMacaulay complexes.

4.4 TheOREM. Let $(\Delta, \pi)$ be a balanced Cohen-Macaulay complex of type $\mathbf{a}=\left(a_{1}, \ldots, a_{m}\right)$, with $\pi=\left(V_{1}, \ldots, V_{m}\right)$. Let $\nu_{i}=$ card $V_{i}$, and let $T$ be a set with $\sum_{i=1}^{m}\left(\nu_{i}-a_{i}\right)$ elements, say $T=\left\{y_{i j}: 1 \leqslant i \leqslant m, 1 \leqslant j \leqslant \nu_{i}-a_{i}\right\}$. Then there exists a multicomplex $\Lambda$ on $T$ with the following property: For every $\left(b_{1}, \ldots, b_{m}\right) \in \mathbf{N}^{m}$, the number of $M \in \Lambda$ satisfying

$$
\sum_{j=1}^{\nu_{i}-a_{i}} M\left(y_{i j}\right)=b_{i}, \text { for all } i \in[m],
$$

is equal to $h_{\mathrm{b}}(\Delta, \pi)$. Hence by (5),

$$
\sum_{j=1}^{\nu_{i}-a_{i}} M\left(y_{i j}\right) \leqslant a_{i}, \quad \text { for all } i \in[m] .
$$

Before proving this result, we first discuss its significance. According to Theorem 1.4, the $h$-vector of a Cohen-Macaulay complex $\Delta$ is the $f$-vector of some multicomplex $\Lambda$. Theorem 4.4 asserts that $\Lambda$ must have certain special properties when $(\Delta, \pi)$ is balanced of type $\left(a_{1}, \ldots, a_{m}\right)$. It follows from (9) that $M\left(y_{i j}\right) \leqslant a_{i}$ for all $i \in[m]$. Thus each $y_{i j} \in T$ has a restriction as to its multiplicity in any $M \in \Lambda$. In general, given a vector $\mathbf{c}=\left(c_{1}, c_{2}, \ldots, c_{r}\right)$ where each $c_{i}$ is a positive integer or $\infty$, there is a characterization analogous to Theorems 1.1 and 1.3 for the $f$-vector of a multicomplex $\Lambda$ on a set $S=\left\{y_{1}, \ldots, y_{r}\right\}$ such that $M\left(y_{i}\right) \leqslant c_{i}$ for all $i \in[r]$. This characterization is essentially due to Clements and Lindström [3], although an explicit numerical statement first appeared in [2] and is restated succinctly in [9]. Note that Theorem 1.1 corresponds to the case $\mathbf{c}=(1,1, \ldots, 1)$ and Theorem 1.3 to the case $c=(\infty, \infty, \ldots, \infty)$. At any rate, Theorem 4.4 shows that $\Lambda$ must satisfy the characterization with $\nu_{i}-a_{i}$ of the $c_{j}$ 's equal to $a_{i}$. But Theorem 4.4 actually asserts a much stronger result, viz., the elements of certain subsets of $T$ cannot have their combined multiplicities greater than $a_{i}$. Moreover, Theorem 4.4 places a restriction not merely on the ordinary $h$-vector of $\Delta$, but on the "refined" numbers $h_{\mathbf{b}}(\Delta, \pi)$. Unfortunately the condition which Theorem 4.4 places on $h_{\mathrm{b}}(\Delta, \pi)$ is not strong enough to characterize the number $h_{\mathrm{b}}(\Delta, \pi)$ when $(\Delta, \pi)$ is balanced of some fixed type $\left(a_{1}, \ldots, a_{m}\right)$ 
unless $m=1$ (Theorem 1.4), so for $m>1$ we do not have as complete a result as Theorem 1.4.

The completely balanced case of Theorem 4.4 is of special interest and deserves a separate statement. When $(\Delta, \pi)$ is completely balanced, (9) implies that each $M\left(y_{i}\right) \leqslant 1$. In other words, $\Lambda$ is actually a complex, not just a multicomplex, and we obtain:

4.5 Corollary. Let $(\Delta, \pi)$ be a completely balanced Cohen-Macaulay complex. Then the $h$-vector of $\Delta$ is the f-vector of some complex $\Lambda$ and therefore satisfies Theorem 1.1. (In other words, $\mathrm{h}(\Delta)$ is not merely an $M$-vector, but also a $K$-vector.) Even more strongly, there is an ordered partition $\left(T_{1}, \ldots, T_{m}\right)$ of the vertex set $T$ of $\Lambda$ such that card $T_{i} \cap F \leqslant 1$ for all $F \in \Lambda, i \in[m]$. Equivalently, the 1-skeleton of $\Lambda$ can be $m$-colored in the usual graph-theoretical sense. Moreover, $\Lambda$ can be chosen so that for any $0-1$ vector $\mathbf{b}=\left(b_{1}, \ldots, b_{m}\right)$, there are exactly $h_{\mathrm{b}}(\Delta, \pi)$ faces $F \in \Lambda$ satisfying: $T_{i} \cap F=\varnothing$ if and only if $b_{i}=0$.

As an example to show that the conditions on $\Lambda$ given by Corollary 4.5 are not sufficient to characterize the $h$-vector of a completely balanced CohenMacaulay complex, let $\Lambda$ be the complex on $T=\left\{x_{1}, \ldots, x_{7}\right\}$ with maximal faces $\left\{x_{1}, x_{2}\right\},\left\{x_{3}, x_{4}\right\},\left\{x_{5}\right\},\left\{x_{6}\right\},\left\{x_{7}\right\}$. Let $T_{1}=\left\{x_{1}, x_{3}, x_{5}, x_{6}, x_{7}\right\}, T_{2}=$ $\left\{x_{2}, x_{4}\right\}$. Then $(\Delta, \pi)$ would satisfy $h_{00}=1, h_{10}=5, h_{01}=2, h_{11}=2$. In particular, $(\Delta, \pi)$ would be of type $(1,1)$ (i.e., a bipartite graph) with 6 vertices and 10 edges, and no such graph exists.

We remark in passing one additional property of completely balanced Cohen-Macaulay complexes $(\Delta, \pi)$. Namely, $\pi$ is uniquely determined up to order. In other words, if $(\Delta, \sigma)$ is also completely balanced, then the entries $V_{i}$ of $\pi$ are a permutation of those of $\sigma$. Thus the 1-skeleton of $\Delta$ is a so-called "uniquely $m$-colorable graph." The proof is omitted. The corresponding statement for arbitrary completely balanced complexes is false, as shown by the example $\Delta=\langle a b, c d\rangle$.

Proof of Theorem 4.4. If $K$ is an extension field of $k$, then the ring $A_{\Delta} \otimes_{k} K$ has the same Hilbert function as $A_{\Delta}$ and is Cohen-Macaulay if and only if $A_{\Delta}$ is Cohen-Macaulay. Hence we may assume $k$ is infinite. By Theorem 4.1 there is an $\mathbf{N}^{m}$-homogeneous system of parameters $\theta_{1}, \ldots, \theta_{d}$ $\left(d=a_{1}+\cdots+a_{m}=1+\operatorname{dim} \Delta\right)$ for $A_{\Delta}$, and our assumption that $k$ is infinite implies we can choose them so that exactly $a_{i}$ of them have degree $\mathbf{e}_{i} \in \mathbf{N}^{m}$. Let $B_{\Delta}=A_{\Delta} /\left(\theta_{1}, \ldots, \theta_{d}\right)$. Hence by (1) and our assumption that $(\Delta, \pi)$ is Cohen-Macaulay, we have

$$
F\left(A_{\Delta}, \lambda\right) \prod_{i=1}^{m}\left(1-\lambda_{i}\right)^{a_{i}}=F\left(B_{\Delta}, \lambda\right) .
$$


Thus by the definition of $h_{\mathbf{b}}(\Delta, \pi)$, we have

$$
F\left(B_{\Delta}, \boldsymbol{\lambda}\right)=\sum_{\mathbf{b}} h_{\mathbf{b}}(\Delta, \pi) \boldsymbol{\lambda}^{\mathbf{b}}
$$

Now suppose $V_{i}=\left\{x_{i 1}, \ldots, x_{i v_{i}}\right\}$. Let $y_{i j}$ denote the image in $B_{\Delta}$ of $x_{i j}$. Since the $a_{i}$ parameters of degree $\mathbf{e}_{\mathbf{i}}$ are linearly independent, it follows that $B_{\Delta}$ is generated as a $k$-algebra by the elements $y_{i j}, 1 \leqslant i \leqslant m, 1 \leqslant j \leqslant \nu_{i}-$ $a_{i}$. Hence $B_{\Delta}$ has a $k$-basis consisting of monomials in these $y_{i j}$. A simple argument due to Macaulay [13] and also given in [24, Theorem 2.1] shows that we can pick this $k$-basis to be a multicomplex $\Lambda$ on the set of $y_{i j}$. By (10), it follows that the number of $M \in \Lambda$ satisfying (8) is $h_{\mathbf{b}}(\Delta, \pi)$, completing the proof.

5. Posets and Möbius functions. In the special case that $\Delta=\Delta(P)$ for some poset $P$, our previous results are closely related to certain well-known concepts associated with $P$. In this section we will sketch this relation.

Let $P$ be a (finite) pure poset with rank function $\rho$, i.e., $\rho(x)$ is the cardinality of a saturated chain of $P$ with maximum element $x$. Let $\hat{P}$ denote the poset obtained by adjoining a minimum element $\hat{0}$ and maximum element $\hat{1}$ to $P$, i.e., $\hat{0}<x<\hat{1}$ for all $x \in P$. Let $\mu$ denote the Möbius function of $\hat{P}$, as defined in [17]. Thus, $\mu$ is a function from $\{(x, y) \in \hat{P} \times \hat{P}: x \leqslant y\}$ to $\mathrm{Z}$ satisfying

$$
\begin{aligned}
\mu(x, x)=1 & \text { for all } x \in \hat{P}, \\
\sum_{x<y<z} \mu(x, y)=0 & \text { for all fixed pairs } x<z \text { in } \hat{P} .
\end{aligned}
$$

We also write $\mu(x)$ for $\mu(\hat{0}, x)$ and $\mu(P)$ for $\mu(\hat{0}, \hat{1})$.

If $\operatorname{dim} P=\delta$ (i.e., every maximal chain of $P$ has cardinality $\delta+1$ ) and $S \subset[\delta+1]$, define $\alpha(P, S)$ to be the number of chains $x_{1}<x_{2}<\cdots<x_{s}$ in $P$ such that $\left\{\rho\left(x_{1}\right), \ldots, \rho\left(x_{s}\right)\right\}=S$. Thus $\alpha(P, \varnothing)=1, \alpha(P,\{i\})$ is the number of elements in $P$ of rank $i$, and $\alpha(P,[\delta+1])$ is the number of maximal chains of $P$. Equivalently, $\alpha(P, S)$ is the number of maximal chains of the poset

$$
P_{S}=\{x \in P: \rho(x) \in S\}
$$

or equivalently, the number of maximal chains of the poset $\hat{P}_{S}$ consisting of $P_{S}$ with $\hat{0}$ and $\hat{1}$ adjoined. Now for $S \subset[\delta+1]$ define

$$
\beta(P, S)=\sum_{T \subset S}(-1)^{\operatorname{card}(S-T)} \alpha(P, T) .
$$

Equivalently, $\alpha(P, S)=\Sigma_{T \subset S} \beta(P, T)$. It is an immediate consequence of "Philip Hall's theorem" [17, Proposition 6] that

$$
\beta(P, S)=(-1)^{1+\operatorname{card} S} \mu\left(P_{S}\right) .
$$


The numbers $\alpha(P, S)$ and $\beta(P, S)$ were studied for various classes of posets in [18], [19], [21], where they were shown to have many interesting properties. For instance, if $\hat{P}$ is a semimodular lattice then $\beta(P, S) \geqslant 0$ for all $S \subset[\delta+$ 1]. It comes as no surprise that this result may be regarded as a consequence of the fact that semimodular lattices are Cohen-Macaulay, and CohenMacaulay posets seem like the right context for obtaining such results.

Many well-known numerical invariants of (pure) posets $\hat{P}$ can be expressed in terms of the basic numbers $\beta(P, S)$. For instance, the "Whitney number of the second kind" $W_{i}(\hat{P})$ is defined by $W_{i}(\hat{P})=\operatorname{card}\{x \in P: \rho(x)=i\}$ and is clearly given by

$$
W_{i}(\hat{P})=\alpha(P,\{i\})=\beta(P,\{i\})+1 .
$$

The "Whitney number of the first kind" $w_{i}(\hat{P})$ is defined by $w_{i}(\hat{P})=$ $\sum_{\rho(x)=i} \mu(x)$, and it is not hard to see that

$$
(-1)^{i} w_{i}(\hat{P})=\beta(P,[i-1])+\beta(P,[i]) .
$$

Another commonly studied invariant is

$$
(-1)^{\delta} \sum_{\rho(x)=i} \mu(x) \mu(x, \hat{1})=\beta(P,[\delta+1]-\{i\})+\beta(P,[\delta+1]) .
$$

A related invariant of $\hat{P}$ is the zeta polynomial [20, §3]. If $m \in \mathbf{N}$, define $Z(P, m)$ to be the number of chains $\hat{0}=x_{0} \leqslant x_{1} \leqslant \cdots \leqslant x_{m}=\hat{1}$ in $\hat{P}$. Thus $Z(P, 0)=0, Z(P, 1)=1$, and $Z(P, 2)=$ card $\hat{P}$. It is easily seen that $Z(P, m)$ is a polynomial function of $m$ of degree $\delta+2$. It follows that there are constants $e_{0}, \ldots, e_{\delta+1}$ and $h_{0}, \ldots, h_{\delta+1}$ such that $Z(P, m)=$ $\sum_{i=1}^{\delta+2} e_{i-1}\left(\begin{array}{c}m \\ i\end{array}\right)$ and

$$
(1-\lambda)^{\delta+3} \sum_{m=0}^{\infty} Z(P, m) \lambda^{m}=\lambda\left(h_{0}+h_{1} \lambda+\cdots+h_{\delta+1} \lambda^{\delta+1}\right) .
$$

It is not hard to see that

$$
e_{i}=\sum_{\substack{S \subset[\delta+1] \\ \operatorname{card} S=i}} \alpha(P, S) \quad \text { and } \quad h_{i}=\sum_{\substack{S \subset[\delta+1] \\ \text { card } S=i}} \beta(P, S) .
$$

We now discuss the relationship between the numbers $\beta(P, S)$ and the complex $\Delta(P)$. We have already noted in $\S 2$ that when $P$ is pure of dimension $\delta$, there is a standard ordered partition $\pi=\left(V_{1}, \ldots, V_{\delta+1}\right)$ defined by $V_{i}=\{x \in P: \rho(x)=i\}$ which makes $(\Delta(P), \pi)$ completely balanced. Now Philip Hall's theorem is equivalent to the formula

$$
\mu(x, y)=\tilde{\chi}(\Delta(x, y)), \quad x<y,
$$

where $(x, y)=\{z \in P: x<z<y\}$ (see [17, p. 346]). In particular, $\mu\left(P_{S}\right)=$ $\tilde{\chi}\left(P_{S}\right)$. It is then an immediate consequence of Proposition 3.5 and (11) (or 
otherwise) that $h_{\mathbf{b}}(\Delta(P), \pi)=\beta(P, S)$, where $\mathbf{b}=\left(b_{1}, \ldots, b_{\delta+1}\right), b_{i}=1$ if $i \in S, b_{i}=0$ if $i \notin S$. Hence we see that if $P$ is a Cohen-Macaulay poset, then the numbers $\beta(P, S)=h_{\mathrm{b}}(\Delta(P), \pi)$ satisfy the stringent requirements of Corollary 4.5. In particular, we obtain new restrictions on the Whitney numbers of the first and second kind of a Cohen-Macaulay poset.

Now note that if $(x, y)$ is an open interval of $P$, then $(x, y)$ is the link of a face of $\Delta(P)$ of the form $x_{1}<x_{2}<\cdots<x_{r}=x<y=y_{s}<y_{s-1}$ $<\cdots<y_{1}$, where $\rho\left(x_{i}\right)=i$ and $\rho\left(y_{j}\right)=\delta+2-j$. Hence by Theorem 1.2, $(x, y)$ is also a Cohen-Macaulay poset. The fact that $\beta(P, S) \geqslant 0$ for any Cohen-Macaulay poset $P$ then implies the following: Let $P$ be a CohenMacaulay poset, and let $(x, y)$ be an open interval of length $l$ in $\hat{P}$. Then $(-1)^{l} \mu(x, y) \geqslant 0$, where $\mu$ is the Möbius function of $\hat{P}$. Indeed, $(-1)^{l} \mu(x, y)$ is the $l$ th Betti number (with respect to the field $k$ ) of the complex $\Delta(x, y)$. In the terminology of poset theory, the Möbius function of $\hat{P}$ "alternates in sign." Since by Theorem 4.3 each $P_{S}$ is Cohen-Macaulay when $P$ is, it follows that the Möbius function of each $P_{S}$ also alternates in sign [23, §8].

It should also be pointed out that when $P$ is any pure poset with the standard ordered partition $\pi$, then the numbers $\alpha(P, S)$ are identical to $f_{\mathrm{b}}(\Delta(P), \pi)$, with $b_{i}=1$ if $i \in S, b_{i}=0$ if $i \notin S$. The zeta polynomial $Z(P, m)$ is just the function $H(\Delta, m-1)$ of (2), and the vector $\left(h_{0}, h_{1}, \ldots, h_{\delta+1}\right)$ of (12) is just the $h$-vector of $\Delta(P)$.

There are two main classes of Cohen-Macaulay posets known: (i) semimodular lattices, or more generally, semimodular posets. (A poset $P$ is semimodular if for every closed interval $I$ of $\hat{P}$, and for every $x, y$ in $I$ such that $x$ and $y$ cover some element $u$ of $I$, there is a $v \in I$ which covers both $x$ and $y$. If $\hat{P}$ is a lattice, then it suffices to consider only the case $I=\hat{P}$.) (ii) The lattice of faces of a regular cell complex (e.g., a simplicial complex or the boundary complex (not necessarily simplicial) of a convex polytope) whose underlying space $X$ satisfies Theorem 1.2(iii). In addition, if $P$ and $Q$ are Cohen-Macaulay, then so is their ordinal sum $P \oplus Q \cdot(P \oplus Q$ is the partial order on the disjoint union of $P$ and $Q$ defined by $x \leqslant y$ in $P \oplus Q$ if (i) $x \leqslant y$ in $P$, or (ii) $x \leqslant y$ in $Q$, or (iii) $x \in P$ and $y \in Q$.) Indeed, $\Delta(P \oplus Q)$ is just the join $\Delta(P) * \Delta(Q)$, and $A_{\Delta(P \oplus Q)}=A_{\Delta(P)} \otimes_{k} A_{\Delta(Q)}$.

When $\hat{P}$ is an admissible lattice there is a combinatorial interpretation of the numbers $\beta(P, S)$ which implies they are nonnegative [21]. To give the reader the flavor of this result, we mention the interpretation of $\beta(P, S)$ when $\hat{P}$ is the lattice of subspaces of an $n$-dimensional vector space over $G F(q)$. In this case,

$$
\beta(P, S)=\sum_{\pi} q^{i(\pi)},
$$


where the sum is over all permutations $\pi=\left(a_{1}, \ldots, a_{n}\right)$ of $[n]$ satisfying $S=\left\{i: a_{i}>a_{i+1}\right\}$, and where $i(\pi)=\operatorname{card}\left\{(i, j): i<j\right.$ and $\left.a_{i}>a_{j}\right\}$. This suggests that admissible lattices are Cohen-Macaulay, and indeed this has been shown by Anders Bjørner (to be published). It would be of considerable interest to obtain results analogous to (13) for other classes of CohenMacaulay posets, such as the semimodular posets which are not lattices, or the lattice of faces of a convex polytope.

\section{REFERENCES}

1. M. F. Atiyah and I. G. Macdonald, Introduction to commutative algebra, Addison-Wesley, Reading, Mass. 1969.

2. G. F. Clements, More on the generalized Macaulay theorem, Discrete Math. 1 (1971), 247-255.

3. G. Clements and B. Lindströ, A generalization of a combinatorial theorem of Macaulay, J. Combinatorial Theory 7 (1969), 230-238.

4. G. Danaraj and V. Klee, Which spheres are shellable?, Ann. Discrete Math. 2 (1978), 33-52.

5. R. Edwards, An amusing reformulation of the Four Color Problem, Notices Amer. Math. Soc. 24 (1977), A-257-A-258. Abstract \#77T-G16.

6. T. Gallai, Transitiv orientierbare Graphen, Acta Math. Acad. Sci. Hungar. 18 (1967), 25-66.

7. A. Ghouilà-Houri, Caractérisation des graphes nonorientés dont on peut orienter les arêtes de manière à obtenir le graphe d'une relation d'ordre, C. R. Acad. Sci. Paris Sér. A-B 254 (1962), 1370-1371.

8. P. Gilmore and A. J. Hoffman, A characterization of comparability graphs and interval graphs, Canad. J. Math. 16 (1967), 539-548.

9. C. Greene and D. J. Kleitman, Proof techniques in the theory of finite sets, MAA Studies in Combinatorics (G.-C. Rota, editor), Math. Assoc. Amer., Washington, D. C. (to appear).

10. M. Hochster, Cohen-Macaulay rings, combinatorics, and simplicial complexes, Proc. Second Oklahoma Ring Theory Conf. (March, 1976), Dekker, New York, 1977.

11. G. Katona, A theorem of finite sets, Proc. Colloq. Theory of Graphs(Tihany, Hungary, 1964, Academic Press, New York; Akad. Kiadó, Budapest, 1968, pp. 187-207.

12. J. B. Kruskal, The number of simplices in a complex, Mathematical Optimization Techniques, Univ. of California Press, Berkeley, Calif., 1963, pp. 251-278.

13. F. S. Macaulay, Some properties of enumeration in the theory of modular systems, Proc. London Math. Soc. 26 (1927), 531-555.

14. P. McMullen, The maximum number of faces of a convex polytope, Mathematika 17 (1970), 179-184.

15. J. Munkres, Topological results in combinatorics (preprint).

16. G. A. Reisner, Cohen-Macaulay quotients of polynomial rings, Advances in Math. 21 (1976), $30-49$.

17. G.-C. Rota, On the foundations of combinatorial theory. I, Theory of Möbius functions, Z. Wahrscheinlichkeitstheorie und Verw. Gebiete 2 (1964), 340-368.

18. R. Stanley, Ordered structures and partitions, Mem. Amer. Math. Soc. No. 119, 1972.

19. ___ Supersolvable lattices, Algebra Universalis 2 (1972), 197-217.

20. __ Combinatorial reciprocity theorems, Advances in Math. 14 (1974), 194-253.

21. __ Finite lattices and Jordan-Hölder sets, Algebra Universalis 4 (1974), 361-371.

22. (1975), 135-142.

23. __ Cohen-Macaulay complexes, Higher Combinatorics (M. Aigner, editor), NATO Advanced Study Institute Series, Reidel, Dordrecht and Boston, 1977, pp. 51-52.

24. , Hilbert functions of graded algebras, Advances in Math. 28 (1978), 57-83. 
25. J. S. Provan, Decompositions, shellings, and diameters of simplicial complexes and convex polyhedra, Thesis, Cornell University, 1977.

Department of Mathematics, Massachusetts Institute of Technology, Cambridge, MASSACHUSETTS 02139

Current address: Department of Mathematics, University of California at San Diego, La Jolla, California 92093 